\title{
The 6 O'clock Anchor Increases Labral Repair Strength in a Biomechanical Shoulder Instability Model
}

\author{
Steven L. Bokshan, MD¹, Steven F. DeFroda, MD, ME${ }^{1}$, Joseph A. Gil, MD1', Rohit Badida, \\ MEng ${ }^{1}$, Joseph J. Crisco, PhD ${ }^{1}$, Brett D. Owens, MD ${ }^{1}$ \\ ${ }^{1}$ Department of Orthopaedic Surgery, Brown University, Warren Alpert School of Medicine, \\ Providence, RI.
}

\begin{abstract}
Background: Arthroscopic Bankart repair is currently the most utilized technique in the surgical management of symptomatic anterior shoulder instability. Despite a growing body of literature regarding optimal repair configurations, little is known about inferior suture anchor placement (6 o'clock position).
\end{abstract}

Methods: 12 cadaveric shoulders were tested on a 6 DOF robotic musculoskeletal simulator to measure the peak resistance force due to anterior displacement of $1 \mathrm{~cm}$. The rotator cuff muscles were loaded during testing to simulate physiological conditions. Test conditions consisted of the intact shoulder, Bankart lesion, Bankart repair (3, 4, and 5 o'clock anchors), and Bankart repair with addition of a 6 o'clock anchor. A 13\% anterior bone defect was then and all conditions were repeated. Repeated measures ANOVA was used to test for significant differences among groups.

Results: In the no bone loss group, the addition of a 6 o'clock anchor yielded the highest peak resistance force $(52.8 \mathrm{~N}, \mathrm{SD}: 4.5 \mathrm{~N})$ and was significantly greater than the standard Bankart repair by $15.8 \%(7.2 \mathrm{~N}, p=0.003)$. With $13 \%$ bone loss from the anterior glenoid, both the standard Bankart repair (peak force 49.3N, SD: 6.1N, $p=0.02$ ) and repair with the addition of the 6 o'clock anchor (peak force $52.6 \mathrm{~N}$, SD: $6.1 \mathrm{~N}, p=0.006$ ) had a significantly higher peak resistance force compared to the bone loss with Bankart lesion group (35.2N, SD: 5.8N). While the 6 o'clock anchor did increase the strength of the standard repair by $6.7 \%$, this was not statistically significant $(p=0.9)$ in the bone loss model.

Conclusions: The addition of a 6 o'clock suture anchor to a "standard" Bankart repair increases the peak resistance force to displacement (no bone loss), although this additional strength is lost with creation of a subcritical anterior glenoid bone defect.

Clinical Relevance: This study provides surgeons with essential biomechanical data to aid in the selection of repair configuration. The six o'clock anchor does not enhance repair strength in a subcritical bone loss model.

Corresponding Author: Steven Bokshan, MD, Department of Orthopaedics, Alpert Medical School of Brown University, 593 Eddy Street, Providence, RI 02903, Phone: 401-444-4030, Fax: 401-444-6182. 


\section{Introduction}

Arthroscopic Bankart repair is currently the most utilized technique in the surgical management of symptomatic shoulder instability. Arthroscopic Bankart repair was first described in 1993, with its popularity rapidly increasing over the past several decades as arthroscopic instrumentation and techniques have improved. ${ }^{1}$ An investigation of the American Board of Orthopaedic Surgery (ABOS) for surgical trends in Bankart repair revealed a stark increase in the percentage of the Bankart repairs done arthroscopically compared to open. ${ }^{2}$ From 2003 to $2005,71.2 \%$ of repairs were performed arthroscopically, compared with $87.7 \%$ from 2006 to 2008 . $^{2}$ With an increasing literature base showing similar clinical outcomes between open and arthroscopic repair, there has been a recent shift towards arthroscopic stabilization as the first-line surgical management of shoulder instability. ${ }^{3-10}$

Despite the growing popularity of arthroscopic Bankart repair, debate remains regarding the optimal repair configuration. ${ }^{11}$ Multiple studies have shown comparable clinical outcomes when comparing knotless to simple stitch suture anchors. ${ }^{12-14}$ Another important consideration is the number of suture anchors. Shibata et al examined the number of suture anchors used during Bankart repair in 102 patients and found that placement of fewer sutures anchors correlated with rucurrence. ${ }^{15}$ Relatively few studies, however, have assessed the specific location on the glenoid with regard to anchor placement and the corresponding biomechanical strength of the construct.

Finally, it is essential to consider the presence of subcritical anterior glenoid bone loss and its effect on repair configuration and strength. While thresholds have been defined ranging from $13.5-17.3 \%$, there is a paucity of literature regarding the effects of various suture anchor configurations in anterior bone loss. ${ }^{16-18}$ This is particularly true for Bankart repairs with the utilization of a 6 o'clock anchor, a rapidly evolving technique in arthroscopic labral surgery. Inferior glenoid anchor placement is believed to enhance the bumper effect of the labrum and to improve shoulder stability by providing a reliable inferior to superior capsular shift during arthroscopic stabilization. Despite this, inferior anchor placement can be particularly challenging based on patient anatomy and surgeon experience. ${ }^{19}$

Despite the current understanding of anterior shoulder instability pathoanatomy, failure rates following arthroscopic Bankart repair remain as high as $14-35 \% .20,21$ The purpose of our study was to characterize the additive effect of a 6 o'clock anchor in the stabilization of a Bankart lesion with and without subcritical glenoid bone loss. We hypothesized that the addition of a 6 o'clock anchor would provide an increased resistance force to anterior displacement when added to a traditional repair utilizing 3, 4, and 5 o'clock anchors. We hypothesized that this additive effect would also remain true with the creation of subcritical anterior glenoid bone loss (13\%). 


\section{Materials and Methods}

\section{Preparation of Specimens}

An a prior power analysis was conducted. With a power level of 0.8 and effect size $f$ of 0.5 , 9 shoulders would be required for a significance level of 0.05 . A total of 12 fresh-frozen cadaver shoulders ( 6 men, 6 women) with an average age of 53.9 years were utilized. Pretesting computerized tomography (CT) scans were performed on all specimen in order to define a reference coordinate system using anatomical features (Figure 1). Specimens were excluded if they had moderate to severe shoulder osteoarthritis based on CT scan or were over the age of 60. All specimens had an intact rotator cuff. All skin and subcutaneous tissues were dissected from the specimen being careful to preserve the rotator cuff muscle bellies and tendons, the long head of the biceps brachii tendon, and the conjoint tendon. Care was made not to destabilize the long head of the biceps from its native groove. To aid in mounting the specimens, a scapular osteotomy was performed along a line parallel to the glenoid surface, and a humeral shaft osteotomy was performed $10 \mathrm{~cm}$ distal to the humeral neck. Both the scapula and humerus were then potted in a two-part urethane compound (300Q, Smooth-On, Macungie, PA) utilizing custom fixtures (Figure 2). The glenoid surface was potted parallel to the horizontal plane, while the humerus was potted in 60 degrees of abduction and neutral rotation. These values were chosen based on previously studied and validated models in the anterior instability literature. ${ }^{16}$ In concordance with previous shoulder instability biomechanical analyses, simulated muscle tension of the rotator cuff was performed by placing $7.5 \mathrm{~N}$ deadweights on the supraspinatus, infraspinatus, teres minor, and subscapularis tendons. ${ }^{1} 10 \mathrm{~N}$ was applied to both the conjoint tendon and the long head of the biceps tendon by utilizing constant force springs.

\section{Testing Apparatus}

A 6 DOF robotic musculoskeletal simulator (KUKA KR 6 R700, Augsburg, Germany) controlled by simVITRO labview software (Cleveland Clinic, Ohio, US) was used in this study. This robot contains a multi-axis load cell (SI-580N, ATI, Apex, NC) for the measurement of forces in all three Cartesian directions. After mounting each specimen, the three dimensional relationships between the robot, load cell, and the specimen were established using a 6-DOF digitizing probe (Optotrak, NDI, CA). A glenoid coordinate system was generated using a digitization of bone-based anatomic landmarks determined from the pre-testing CT scan. In addition to the loaded rotator cuff, a $5 \mathrm{~N}$ compressive force was applied to the humerus during testing in order to to keep the humeral head centered similar to previously validated models. ${ }^{16}$ Starting from the centered position, the humeral head was displaced $1 \mathrm{~cm}$ in the anterior direction at a rate of $1.0 \mathrm{~mm} / \mathrm{sec}$. The peak force against the humeral head during anterior displacement was recorded. Two trials were performed for each test condition and the mean value was used for data analysis.

\section{Test Conditions}

The sequence of test conditions was fixed and consisted of: (1) intact shoulder, (2) simulated anterior-inferior labral (Bankart) lesion by detaching the labrum from the 3 to 6 o'clock positions, (3) Bankart repair using suture anchors at the 3,4, and 5 o'clock positions, (4) and Bankart repair (3,4, and 5 o'clock anchors) with the addition of a 6 o'clock suture anchor 
(Figure 3). An anterior subcritical bone loss model was then created by removing $13 \%$ of the anterior to posterior glenoid width (measured on CT scan) in a plane perpendicular to the long axis of the glenoid. ${ }^{16}$ Conditions (2), (3), and (4) were then repeated using this bone loss model. In order to access the labrum, a capsulotomy was made from the 2-7 o'clock positions and subsequently repaired after the labral repair. Gryphon ${ }^{\mathrm{TM}}$ BR Suture Anchors were provided courtesy of DePuy Synthes (Raynham, MA).

\section{Statistical Analysis}

A one-way repeated-measures analysis of variance (RM-ANOVA) was used to determine the difference in mean peak force values for both the intact and bone loss groups. Post-hoc Dunnett analyses were performed to examine significant differences among each individual group. A $p$-value of 0.05 was used to determine significance for all tests (SPSS Statistics V21.0, IBM Corporation, Armonk, NY, USA).

\section{Source of Funding}

Funding of this project was provided in part by DePuy Synthes (Raynham, MA). Research reported in this publication was supported by the National Institute of Arthritis and Musculoskeletal and Skin Diseases of the National Institutes of Health under Award Number P30GM12273 (COBRE Bio-engineering Core). The content is solely the responsibility of the authors and does not necessarily represent the official views of the National Institutes of Health.

\section{Results}

\section{No Bone Loss}

The average peak resistance force to anterior displacement in the intact shoulder group was 43.3N (SD: $15.7 \mathrm{~N}$ ). Upon cutting the labrum to create a Bankart lesion, there was a statistically significant decrease in resistance force $(34.4 \mathrm{~N}, \mathrm{SD}: 18.6 \mathrm{~N}, p=0.001)$. The peak resistance force of the Bankart repair group with suture anchors at 3,4, and 5 o' clock was not statistically different from that intact shoulder group $(p=0.99)$. The addition of a 6 o'clock anchor yielded the highest peak resistance force $(52.8 \mathrm{~N}, \mathrm{SD}: 4.5 \mathrm{~N})$ and was significantly greater (Figure 4a) than the standard Bankart repair by $15.8 \%(7.2 \mathrm{~N}, p=$ 0.003). The peak force for both the standard Bankart repair $(p=0.02)$ and the 6 o'clock anchor group $(p=0.001)$ were significantly greater than the Bankart lesion group.

\section{$13 \%$ Subcritical Bone Loss}

Both the standard Bankart repair (peak force 49.3N, SD: $6.1 \mathrm{~N}, p=0.02$ ) and repair with the addition of the 6 o'clock anchor (peak force $52.6 \mathrm{~N}, \mathrm{SD}: 6.1 \mathrm{~N}, p=0.006$ ) had a significantly greater peak resistance force (Figure $4 \mathrm{~b}$ ) compared to the subcritical bone loss with Bankart lesion group $(35.2 \mathrm{~N}, \mathrm{SD}: 5.8 \mathrm{~N})$. While the 6 o' clock anchor did increase the strength of the standard repair by $6.7 \%$ on average, this increase was not statistically significant $(p=0.9)$ 


\section{Discussion}

Our study found that the addition of a 6 o'clock anchor to a standard Bankart configuration significantly increased the peak resistance force with anterior humeral head displacement in a shoulder instability model. The addition of a 6 o'clock anchor tended to increase the peak resistance force when a $13 \%$ subcritical bone defect was created, however this value was not statistically significant. These findings are important to consider when attempting to perform Bankart repair with the optimal construct. While placement of the 6 o'clock anchor can be difficult depending on surgeon experience, patient position, patient anatomy, and portal location, the above findings indicate that this anchor may enhance repair strength during Bankart repair in the absence of subcritical bone loss. Ultimately, the 6 o'clock anchor may achieve this additive effect by assisting with a "capsular shift" during the repair, i.e. shifting the capsulo-labral tissue of the glenoid superiorly by $1 / 2$ o' clock. ${ }^{22}$ Despite these benefits, surgeons must be aware of the risk to the surrounding axillary nerve when using this anchor placement. ${ }^{19,22,23}$

Optimal suture anchor placement and configuration for Bankart repair continues to be investigated. ${ }^{24-27}$ Many biomechanical studies focus on restoration of the capsulo-labral footprint as well as load to failure of the construct. Judson et al performed an anatomic and biomechanical study to determine differences in footprint restoration and mechanical properties for four suture configurations: single row with simple sutures, single row with horizontal sutures, double row with suture, double row with labral tape. ${ }^{28}$ It was reported that double row repair restored more of the anatomic footprint than the single row techniques, however mechanical properties were not different among the two groups. ${ }^{28}$ More relevant to our study however was anchor placement. For single row repairs, the authors placed anchors at the articular margin of the glenoid at the 2:30, 4, and 5-o'clock positions. Double row anchors were placed in a "W" configuration with medial anchors placed at the 3-o'clock and 4:30 positions on the medial border of the labral footprint on the medial glenoid and the lateral anchors placed at the 2:30, 4, and 5-o'clock positions. ${ }^{28}$ It is important to note that the authors did not investigate the usage of the 6 o'clock anchor, nor did they test for resistance to displacement, but rather load to failure of the repair construct. These authors do not find any differences in load to failure among different repair configurations.

Bankart repair is reliant on restoring native anatomy as well as decreasing capsular volume and redundancy. Repair of the glenoid labrum also produces a bumper effect, which can resist excessive anterior translation of the humeral head. It is for these reasons that we believe the $6 \mathrm{o}$ 'clock anchor increases the force required for anterior humeral displacement in our shoulder model. Placement of an additional anchor at the 6 o'clock position restores the labral bumper more so than a traditional repair with the most inferior anchor being placed at 5 o'clock or 5:30. As the inferior most aspect of the Bankart lesion typically occurs at the 6 o'clock position, mobilization of the tissue at this position may aid in decreasing capsular volume while allowing for capsulolabral tissue to be turned towards the face of the glenoid in order to serve as a restraint to anterior translation. ${ }^{22,29}$ Natera et al describe a single anchor purse string technique which is based on this theory. ${ }^{29}$ The authors place a single anchor at the 4 o'clock position and then one limb of the suture is passed through the 
capsulolabral tissue at the 6 o'clock position while the other limb is passed at the 2 o'clock position. The two limbs are tied down to allow for mobilization of the labral tissue superiorly, creating an anterior capsulolabral bumper to resist translation. ${ }^{29}$ Good clinical results have been reported with this technique, with 37 shoulders with mean 3-year follow up reporting only a 5.4\% recurrent instability rate, and a $97 \%$ return to sport, with $66 \%$ return to pre-injury activity level. ${ }^{30}$

While our study showed a significant increase in resistance to displacement force for a Bankart lesion model, the same was not true when considering specimens with subcritical anterior bone loss. The bony architecture of the glenohumeral joint serves as an important static restraint. Loss of glenoid bone can lead to altered humeral head kinematics by altering the glenoid track. Shin et al performed a biomechanical study to determine the amount of bone loss at which soft tissue repair was no longer adequate to restore anterior-inferior glenohumeral translation. ${ }^{17}$ The authors performed serial osteotomies to examine the shoulder with $10 \%, 15 \%, 20 \%$, and $25 \%$ glenoid bone defects and found that glenohumeral translation could not be adequately restored once the boney defect exceeded $15 \% .{ }^{17} \mathrm{It}$ is worth noting that this study repaired the labrum with 3 anchors in the 3-o'clock, 4-o'clock, and 5:30 positions. ${ }^{17}$ Contrary to this study, an isolated soft tissue Bankart repair was able to restore the native force in a $13 \%$ glenoid bone loss model. While both the "traditional repair configuration" and 6 o'clock anchor restored peak translational force compared to the injured state, the addition of a 6 o'clock anchor did not provide additional stability in the glenoid bone loss model. We postulate that this demonstrates the combined importance of both boney and soft tissue restraint. Determination of the maximal amount of bone loss at which the 6 o'clock anchor offers a significant increase in translational resistance is an area of potential future study.

This study has several limitations. First, shoulder instability is a dynamic event which relies on the complex interplay between bony stabilization as well as dynamic stabilization from the rotator cuff musculature. We attempted to best simulate horizontal compression of the humeral head into the glenoid by applying a constant compressive force, loading the rotator cuff muscles, long head of the biceps, and conjoint tendon with weights, however this may not have been an exact replicate of physiological conditions. Additionally, we applied forces with the humeral head in a neutral position which may not be the true vector of force experienced during dislocation. This testing method has been previously used and its limitations discussed in prior publications. ${ }^{31}$ Additionally, placement of the 6 o'clock anchor has been shown to be exceedingly difficult during procedures, with a high rate of suture anchor cortical perforation, which can lead to weakness of the repair and ultimate clinical failure. ${ }^{32}$ In our study, anchors were placed in an open fashion. While this made anchor placement highly reliable, is not realistic in a clinical setting and does not address the technical challenges of accurate anchor placement. Despite the aforementioned limitations, this study demonstrated the biomechanical advantages of the 6 o'clock anchor with regards to the prevention of anterior instability. 


\section{Conclusion}

In this study, the addition of a 6 o'clock suture anchor to a "standard" Bankart repair increased the peak resistance to displacement force (no bone loss), although this additional strength was lost with creation of a $13 \%$ anterior glenoid bone defect. Given the growing popularity of arthroscopic Bankart repair and evolving knowledge of the importance of glenoid bone loss for anterior shoulder instability, this study provides surgeons with essential biomechanical data to select the best repair configuration on an individual patient basis. In addition to the proposed benefits of added stability, surgeons must be aware of the technical challenges of inferior anchor placement with regard to patient anatomy. Future work is needed to completely understand the strength of Bankart repair configurations.

\section{Acknowledgments}

Funding of this project was provided in part by DePuy Synthes (Raynham, MA). Dr. Owens is a consultant for Mitek, and MTF/CONMED. Research reported in this publication was supported by the National Institute of Arthritis and Musculoskeletal and Skin Diseases of the National Institutes of Health under Award Number P30GM12273 (COBRE Bio-engineering Core). The content is solely the responsibility of the authors and does not necessarily represent the official views of the National Institutes of Health.

\section{References}

1. el Akad AM, Winge S, Molinari M, Eriksson E. Arthroscopic Bankart procedures for anterior shoulder instability. A review of the literature. Knee Surg Sports Traumatol Arthrosc. 1993;1(2):113-122. http://www.ncbi.nlm.nih.gov/pubmed/8536006. Accessed May 5, 2017. [PubMed: 8536006]

2. Owens BD, Harrast JJ, Hurwitz SR, Thompson TL, Wolf JM. Surgical trends in Bankart repair: an analysis of data from the American Board of Orthopaedic Surgery certification examination. Am J Sports Med. 2011;39(9):1865-1869. doi:10.1177/0363546511406869. [PubMed: 21628637]

3. Aboalata M, Plath JE, Seppel G, Juretzko J, Vogt S, Imhoff AB. Results of Arthroscopic Bankart Repair for Anterior-Inferior Shoulder Instability at 13-Year Follow-up. Am J Sports Med. 2016:1-6. doi:10.1177/0363546516675145.

4. Alkaduhimi H, van der Linde JA, Willigenburg NW, Paulino Pereira NR, van Deurzen DFP, van den Bekerom MPJ. Redislocation risk after an arthroscopic Bankart procedure in collision athletes: a systematic review. J Shoulder Elb Surg. 2016;25(9):1549-1558. doi:10.1016/j.jse.2016.05.002.

5. Blonna D, Bellato E, Caranzano F, Assom M, Rossi R, Castoldi F. Arthroscopic Bankart Repair Versus Open Bristow-Latarjet for Shoulder Instability: A Matched-Pair Multicenter Study Focused on Return to Sport. Am J Sports Med. 2016;44(12):3198-3205. doi:10.1177/0363546516658037. [PubMed: 27501835]

6. Carreira DS, Mazzocca AD, Oryhon J, Brown FM, Hayden JK, Romeo AA. A prospective outcome evaluation of arthroscopic Bankart repairs: minimum 2-year follow-up. Am J Sports Med. 2006;34(5):771-777. doi:10.1177/0363546505283259. [PubMed: 16627629]

7. Green MR, Christensen KP. Arthroscopic versus open Bankart procedures: a comparison of early morbidity and complications. Arthroscopy. 1993;9(4):371-374. http://www.ncbi.nlm.nih.gov/ pubmed/8216566. Accessed February 21, 2017. [PubMed: 8216566]

8. Guanche CA, Quick DC, Sodergren KM, Buss DD. Arthroscopic Versus Open Reconstruction of the Shoulder in Patients with Isolated Bankart Lesions. Am J Sports Med. 1996;24(2):144-148. doi:10.1177/036354659602400204. [PubMed: 8775110]

9. Virk MS, Manzo RL, Cote M, et al. Comparison of Time to Recurrence of Instability After Open and Arthroscopic Bankart Repair Techniques. Orthop J Sport Med. 2016;4(6):2325967116654114. doi:10.1177/2325967116654114. 
10. Wang L, Liu Y, Su X, Liu S. A Meta-Analysis of Arthroscopic versus Open Repair for Treatment of Bankart Lesions in the Shoulder. Med Sci Monit. 2015;21:3028-3035. doi:10.12659/ MSM.894346. [PubMed: 26446430]

11. DeFroda S, Bokshan S, Stern E, Sullivan K, Owens BD. Arthroscopic Bankart Repair for the Management of Anterior Shoulder Instability: Indications and Outcomes. Curr Rev Musculoskelet Med. 2017;10(4):442-451. doi:10.1007/s12178-017-9435-2. [PubMed: 28971317]

12. Thal R, Nofziger M, Bridges M, Kim JJ. Arthroscopic Bankart Repair Using Knotless or BioKnotless Suture Anchors: 2- to 7-Year Results. Arthrosc J Arthrosc Relat Surg. 2007;23(4):367-375. doi:10.1016/j.arthro.2006.11.024.

13. Hayashida K, Yoneda M, Mizuno N, Fukushima S, Nakagawa S. Arthroscopic Bankart repair with knotless suture anchor for traumatic anterior shoulder instability: results of short-term follow-up. Arthroscopy. 2006;22(6):620-626. doi:10.1016/j.arthro.2006.03.006. [PubMed: 16762700]

14. Garofalo R, Mocci A, Moretti B, et al. Arthroscopic Treatment of Anterior Shoulder Instability Using Knotless Suture Anchors. Arthrosc J Arthrosc Relat Surg. 2005;21(11):1283-1289. doi:10.1016/j.arthro.2005.08.033.

15. Shibata H, Gotoh M, Mitsui Y, et al. Risk factors for shoulder re-dislocation after arthroscopic Bankart repair. J Orthop Surg Res. 2014;9(1):53. doi:10.1186/s13018-014-0053-z. [PubMed: 24993404]

16. Shin S-J, Kim RG, Jeon YS, Kwon TH. Critical Value of Anterior Glenoid Bone Loss That Leads to Recurrent Glenohumeral Instability After Arthroscopic Bankart Repair. Am J Sports Med. 2017;45(9):1975-1981. doi:10.1177/0363546517697963. [PubMed: 28333542]

17. Shin S-J, Koh YW, Bui C, et al. What Is the Critical Value of Glenoid Bone Loss at Which Soft Tissue Bankart Repair Does Not Restore Glenohumeral Translation, Restricts Range of Motion, and Leads to Abnormal Humeral Head Position? Am J Sports Med. 2016;44(11):2784-2791. doi:10.1177/0363546516656367. [PubMed: 27480979]

18. Dickens JF, Owens BD, Cameron KL, et al. The Effect of Subcritical Bone Loss and Exposure on Recurrent Instability After Arthroscopic Bankart Repair in Intercollegiate American Football. Am J Sports Med. 2017;45(8):1769-1775. doi:10.1177/0363546517704184. [PubMed: 28474965]

19. Frank RM, Mall NA, Gupta D, et al. Inferior Suture Anchor Placement During Arthroscopic Bankart Repair. Am J Sports Med. 2014;42(5):1182-1189. doi:10.1177/0363546514523722. [PubMed: 24576744]

20. Randelli P, Ragone V, Carminati S, Cabitza P. Risk factors for recurrence after Bankart repair a systematic review. Knee Surgery, Sport Traumatol Arthrosc. 2012;20(11):2129-2138. doi:10.1007/ s00167-012-2140-1.

21. Owens BD, DeBerardino TM, Nelson BJ, et al. Long-term follow-up of acute arthroscopic Bankart repair for initial anterior shoulder dislocations in young athletes. Am J Sports Med. 2009;37(4):669-673. doi:10.1177/0363546508328416. [PubMed: 19218560]

22. Seroyer ST, Nho SJ, Provencher MT, Romeo AA. Four-quadrant approach to capsulolabral repair: an arthroscopic road map to the glenoid. Arthroscopy. 2010;26(4):555-562. doi:10.1016/ j.arthro.2009.09.019. [PubMed: 20362838]

23. Cvetanovich GL, McCormick F, Erickson BJ, et al. The Posterolateral Portal: Optimizing Anchor Placement and Labral Repair at the Inferior Glenoid. Arthrosc Tech. 2013;2(3):e201-e204. doi:10.1016/J.EATS.2013.02.011. [PubMed: 24265983]

24. Ahmed I, Ashton F, Robinson CM. Arthroscopic Bankart Repair and Capsular Shift for Recurrent Anterior Shoulder Instability. J Bone Jt Surg. 2012;94(14):1308-1315. doi:10.2106/JBJS.J.01983.

25. Ahmed I, Ashton F, Robinson CM. Arthroscopic Bankart repair and capsular shift for recurrent anterior shoulder instability: functional outcomes and identification of risk factors for recurrence. $\mathrm{J}$ Bone Joint Surg Am. 2012;94(14):1308-1315. doi:10.2106/JBJS.J.01983. [PubMed: 22810402]

26. Ahmad CS, Galano GJ, Vorys GC, Covey AS, Gardner TR, Levine WN. Evaluation of glenoid capsulolabral complex insertional anatomy and restoration with single- and double-row capsulolabral repairs. J Shoulder Elb Surg. 2009;18(6):948-954. doi:10.1016/j.jse.2009.03.022.

27. Castagna A, Markopoulos N, Conti M, Rose GD, Papadakou E, Garofalo R. Arthroscopic Bankart Suture-Anchor Repair: Radiological and Clinical Outcome at Minimum 10 Years of Follow-up. 
Am J Sports Med. 2010;38(10):2012-2016. doi:10.1177/0363546510372614. [PubMed: 20595552]

28. Judson CH, Voss A, Obopilwe E, Dyrna F, Arciero RA, Shea KP. An Anatomic and Biomechanical Comparison of Bankart Repair Configurations. Am J Sports Med. 2017;45(13):3004-3009. doi:10.1177/0363546517717671. [PubMed: 28777665]

29. Natera LG, Consigliere P, Witney-Lagen C, et al. The Purse String; Procedure for Recurrent Anterior Glenohumeral Instability: A Simple Technique to Achieve Bankart Repair, Capsular Shift, and a Good Labral Bumper. Arthrosc Tech. 2017;6(4):e1245-e1251. doi:10.1016/ j.eats.2017.04.015. [PubMed: 29354424]

30. Levy O, Matthews T, Even T. The purse-string; technique: an arthroscopic technique for stabilization of anteroinferior instability of the shoulder with early and medium-term results. Arthroscopy. 2007;23(1):57-64. doi:10.1016/j.arthro.2006.10.006. [PubMed: 17210428]

31. Nacca C, Gil JA, DeFroda SF, Badida R, Owens BD. Comparison of a Distal Tibial Allograft and Scapular Spinal Autograft for Posterior Shoulder Instability With Glenoid Bone Loss. Orthop J Sport Med. 2018;6(7):232596711878669. doi:10.1177/2325967118786697.

32. Lim TK, Koh KH, Lee SH, et al. Inferior Anchor Cortical Perforation With Arthroscopic Bankart Repair: A Cadaveric Study. Arthrosc J Arthrosc Relat Surg. 2013;29(1):31-36. doi:10.1016/ j.arthro.2012.08.013. 


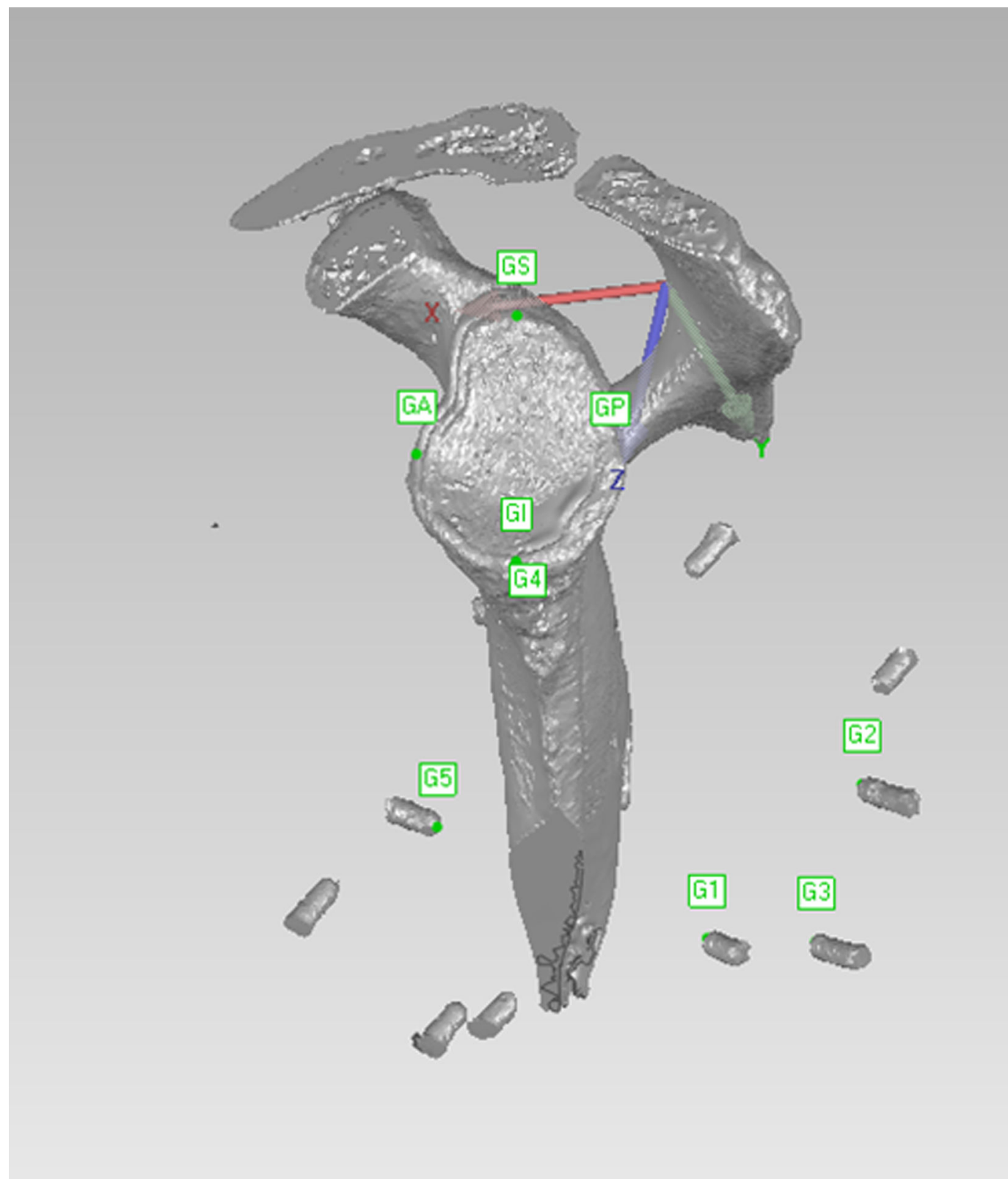

Figure 1:

Three dimensional glenoid coordinate system used to calibrate the robotic arm based on pretest CT scanning. 


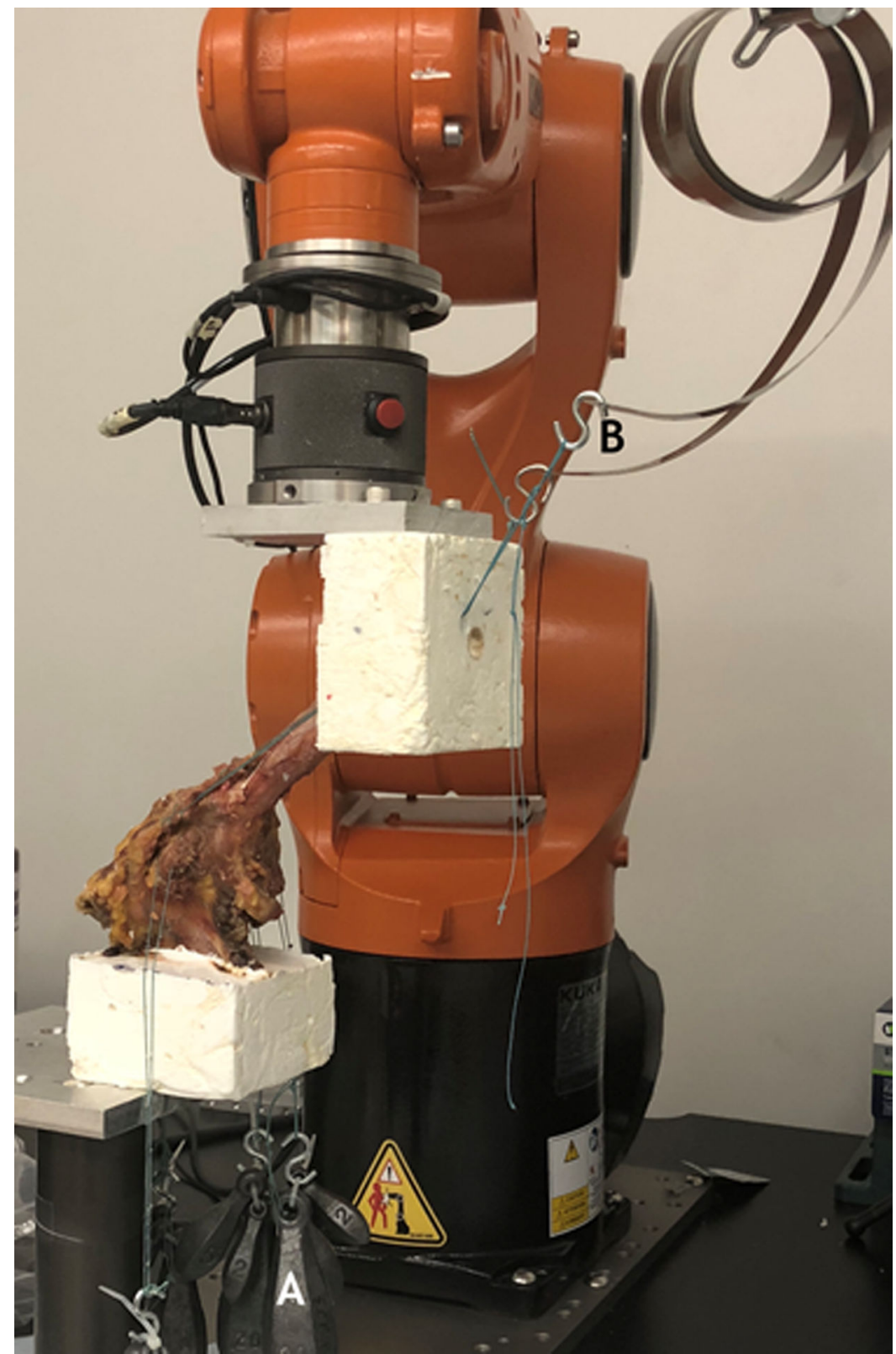

Figure 2:

Robotic arm (GmbH, Ausburg, Germany) with mounted specimen. Loading of the rotator cuff was performed using deadweights (A, rotator cuff) and constant force springs (B, conjoint and biceps tendons). 


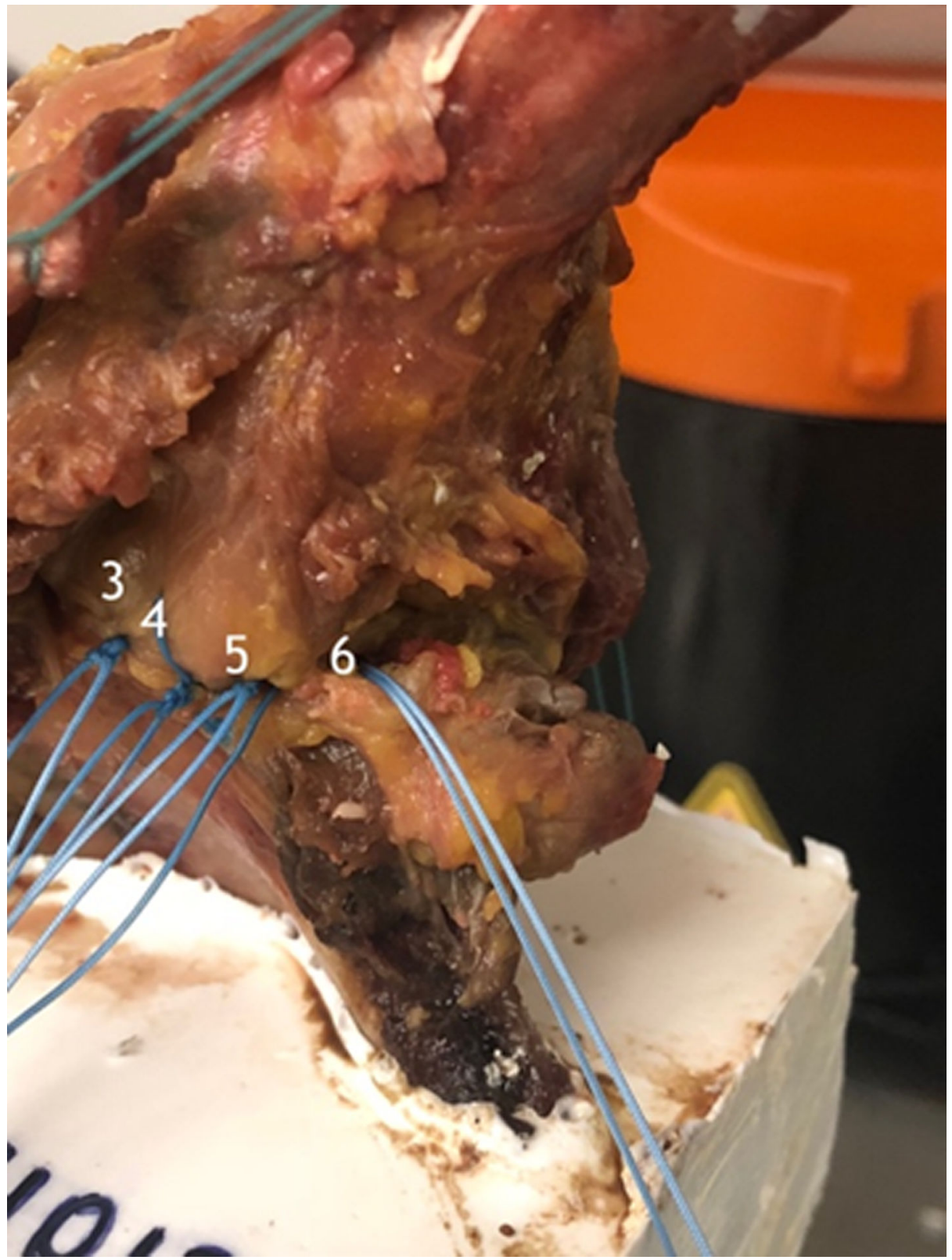

Figure 3:

Suture anchors for the " 6 o'clock" test condition were placed at the standard 3,4, and 5 o'clock positions in addition to the most inferior portion of the glenoid ( 6 o'clock). 


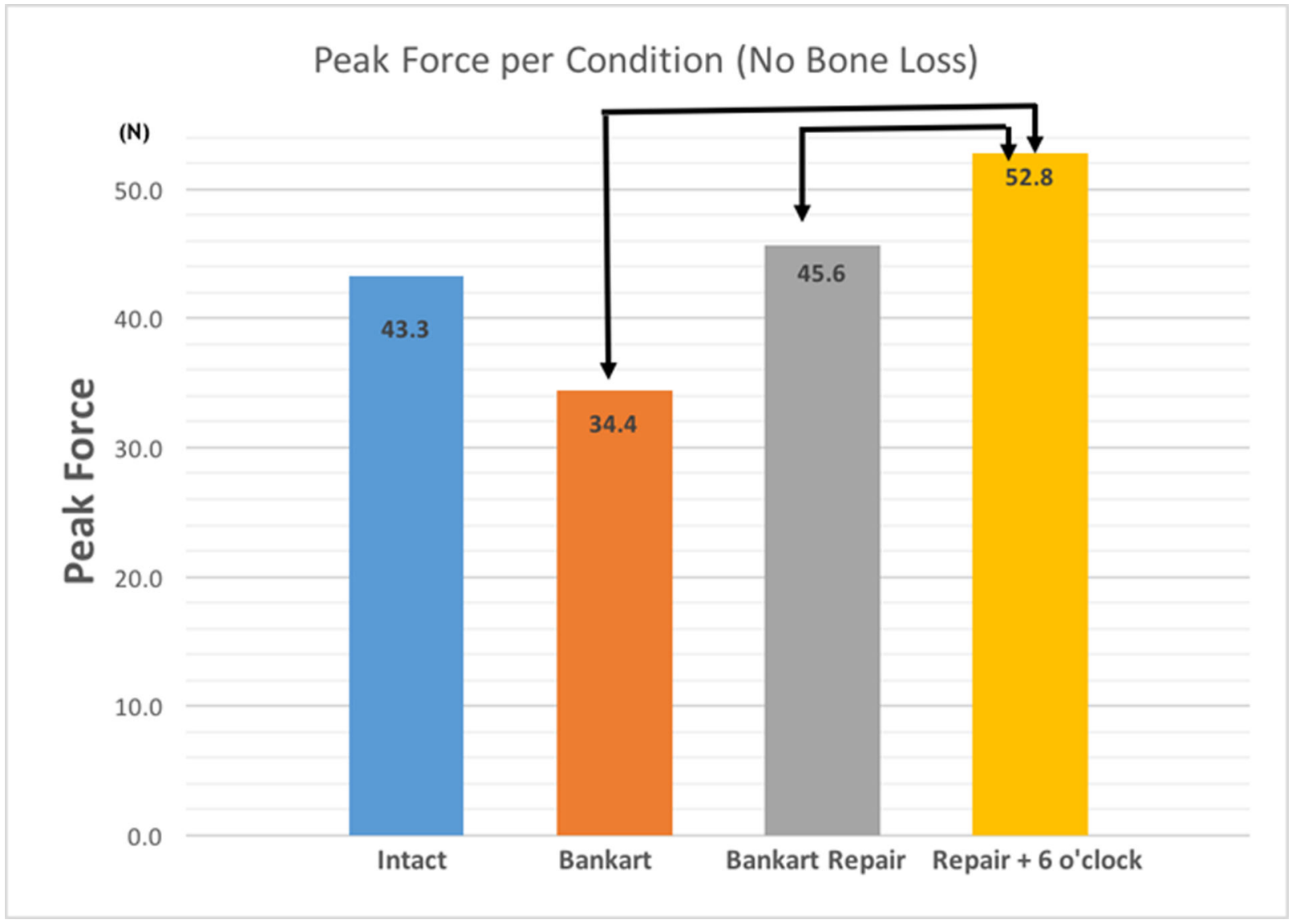

Figure 4a:

The 6 o'clock anchor group provides a higher resistance to displacement compared to the standard Bankart repair as well as compared to Bankart lesion. Arrows denote statistical significance. 


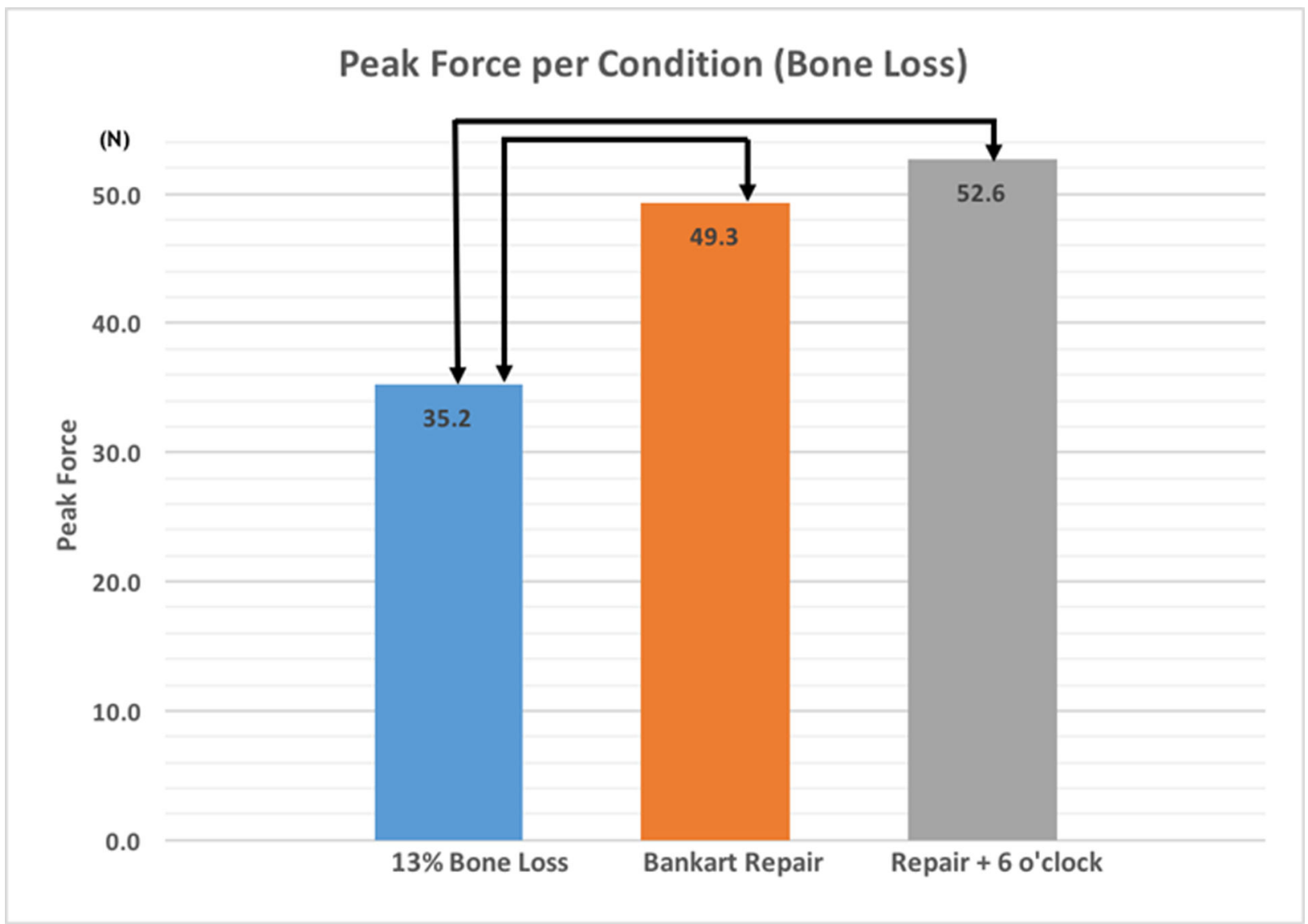

Figure 4b:

The 6 o'clock anchor group did not provide a higher resistance to displacement in the $13 \%$ bone loss model compared to the standard Bankart repair. Both repaired conditions were statistically greater than the Bankart with subcritical bone loss group. Arrows denote statistical significance. 